\title{
Kein Einfluss auf die Progression?
}

Fragestellung: Besteht ein Zusammenhang zwischen einer Interferon-beta-Behandlung und einer Veränderung der Behinderungsprogression bei Patienten mit schubförmiger Multipler Sklerose (MS)?

Hintergrund: Zahlreiche Studien konnten anhand kernspintomografischer sowie klinischer Parameter einen Behandlungsvorteil von Interferon-beta gegenüber Placebo nachweisen. Als klinischer Endpunkt ist insbesondere die Reduktion der jährlichen Schubrate verwendet worden. Ein wesentlich härterer Parameter ist jedoch die Reduktion der Erkrankungsprogression oder auch die Verbesserung des Behinderungsgrades. Gegenstand langjähriger Diskussionen war daher die Frage, welche Parameter tatsächlich von klinischer Relevanz sind. Welche Bedeutung hat in diesem Zusammenhang beispielsweise die Reduktion der jährlichen Schubrate, wenn der Grad der Behinderung trotzdem voranschreitet und ist unter diesen Gesichtspunkten die Reduktion der Erkrankungsprogression nicht der klinisch sinnvollste Parameter? Um die Wirkung der Interferon-Präparate im Hinblick auf die Reduktion der Erkrankungsprogression besser beurteilen zu können, hat eine kanadische Arbeitsgruppe retrospektiv ihre Patientendaten der letzten 23 Jahre untersucht.

Patienten und Methodik: Es handelt sich um eine retrospektive Kohortenstudie, die auf prospektiv erhobenen Daten von 1985 bis 2008 in British Columbia, Kanada, basiert. Patienten mit einer schubförmig verlaufenden MS, die mit einem Interferon-betaPräparat behandelt wurden $(\mathrm{n}=868)$, wurden mit unbehandelten Patienten $(n=829)$ aus dem gleichen Zeitraum sowie mit historischen Kohorten $(n=959)$ verglichen. Primäres Outcome-Kriterium war die Zeit der initialen Interferon-Behandlung (Baseline) bis zu einem bestätigten und beständigen EDSS-Score von 6 (Notwendigkeit einer einseitigen Gehhilfe um $100 \mathrm{~m}$ zu gehen). Im Rahmen eines multivarianten Cox-Regressions-Modells wurden die einzelnen Gruppen miteinander verglichen.

Ergebnisse: Die mittlere aktive Follow-up-Zeit in den verschiedenen Kohorten war 5,1 Jahre für die Interferon behandelten Patienten, 4,0 Jahre für die unbehandelte Therapiegruppe sowie 10,8 Jahre für die historischen Kontrollen. Die beobachtete Rate von Patienten, die einen EDSS-Score von 6 erreichten, war $10,8 \%, 5,3 \%$ sowie $23,1 \%$ für die drei Kohorten (Interferon-Behandlung, unbehandelte Gruppe, historische Kontrollen). Nach einer Adjustierung für potenzielle Baseline-Ungleichheiten $(\mathrm{Ge}$ schlecht, Alter, Erkrankungsdauer sowie initialer EDSS-Score)

Shirani A, ZhaoY, Karim ME et al. Association between use of interferon beta and progression of disability in patients with relapsing-remitting multiple sclerosis. JAMA 2012; 308: $247-56$ zeigten die Patienten unter Interferon-Behandlung kein

Patienten oder Komorbiditäten einbezogen, änderten nichts an den fehlenden Unterschieden zwischen den Gruppen.

Schlussfolgerung: Die Autoren folgern, dass eine Behandlung mit Interferon-beta-Präparaten bei Patienten mit schubförmig verlaufender MS nicht mit einer Reduktion der Erkrankungsprogression assoziiert ist.

\section{-Kommentar von Prof. Volker Limmroth Unnötige Unruhe}

Die Arbeit stellt eine sehr provokante Hypothese auf. Sie widerspricht vielen klinischen Studien mit Interferon-Präparaten, die zwar zum Teil nur geringe Reduktionen der Erkrankungsprogression, aber doch eindeutig statistisch signifikante Unterschiede zu Placebo aufzeigten. Das Problem beim Vergleich sehr unterschiedlicher heterogener Patientengruppen im Hinblick auf harte, schwer bewegliche Parameter liegt darin, dass nur Mittelwerte mit großen Standardabweichungen gerechnet werden, die dann aufgrund der großen Standardabweichungen statistisch nicht mehr unterschiedlich sind.

Insbesondere der Vergleich mit unbehandelten Gruppen bleibt fragwürdig, weil es für die Nichtbehandlung dieser $\mathrm{Pa}$ tienten meist gute Gründe gab. Entweder waren sie in einer Situation, in der eine Behandlung klinisch für noch nicht notwendig gehalten wurde (Gefahr der Selektion übermäßig vieler Patienten mit benignem Krankheitsverlauf), oder dass Patientinnen aufgrund von zahlreichen Schwangerschaften nicht behandelt werden durften (zahlreiche Schwangerschaften können den Erkrankungsverlauf ebenfalls therapeutisch beeinflussen). Seltener dürfte der Fall vorgelegen haben, dass Patienten sich grundsätzlich nicht mit Medikamenten behandeln lassen wollten. Die Schwäche der Arbeit liegt genau hier, in den fehlenden Angaben, warum die unbehandelte Gruppe nicht behandelt worden ist.

Die Daten dieser Arbeit sind somit zwar interessant, aber die Autoren können ihre Hypothese aufgrund der fehlenden Gruppencharakterisierungen nicht gut belegen. Insofern bringt die Arbeit unnötigerweise Unruhe in die MS-Behandlung.

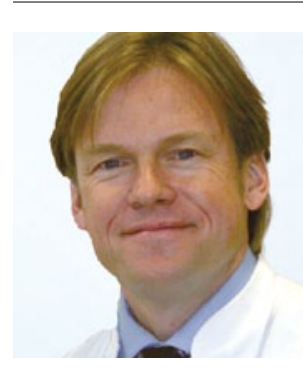

Prof. Dr. med. Volker Limmroth, Köln-Merheim einen EDSS-Score von $6 \mathrm{zu}$ entwickeln als die beiden anderen Gruppen.

Auch weitere Subgruppenanalysen, die den sozioökonomischen Hintergrund der
Chefarzt der Klinik für Neurologie und
Palliativmedizin Köln-Merheim
E-Mail: LimmrothV@kliniken-koeln.de 